\title{
BMJ Open Associations of subclinical heart failure and atrial fibrillation with mild cognitive impairment: a cross-sectional study in a subclinical heart failure screening programme
}

\author{
Elizabeth L Potter (1) , ${ }^{1,2}$ Satish Ramkumar, ${ }^{1,2}$ Leah Wright, ${ }^{1}$ Thomas H Marwick ${ }^{1,2}$
}

To cite: Potter EL, Ramkumar S, Wright $L$, et al. Associations of subclinical heart failure and atrial fibrillation with mild cognitive impairment: a crosssectional study in a subclinical heart failure screening programme. BMJ Open 2021;11:e045896. doi:10.1136/ bmjopen-2020-045896

- Prepublication history and additional supplemental material for this paper are available online. To view these files, please visit the journal online (http://dx.doi.org/10.1136/ bmjopen-2020-045896).

Received 15 0ctober 2020 Accepted 28 May 2021

Check for updates

(C) Author(s) (or their employer(s)) 2021. Re-use permitted under CC BY-NC. No commercial re-use. See rights and permissions. Published by BMJ.

${ }^{1}$ Imaging Research, Baker Heart and Diabetes Institute, Melbourne, Victoria, Australia ${ }^{2}$ School of Public Health and Preventive Medicine, Monash University, Melbourne, Victoria, Australia

Correspondence to

Dr Elizabeth L Potter;

Liz.Potter@baker.edu.au

\section{ABSTRACT}

Objectives Effective identification and management of subclinical left ventricular (LV) dysfunction (LVD) and subclinical atrial fibrillation (AF) by screening elderly populations might be compromised by mild cognitive impairment (MCl). We sought to characterise the prevalence and profile of $\mathrm{MCl}$ and evaluate associations with LV and left atrial $(L A)$ dysfunction and $A F$, in a trial of screening for subclinical LVD and AF.

Design Cross-sectional.

Setting Australian, community-based intervention trial. Participants Adults aged $\geq 65$ years with $\geq 1$ LVD risk factors without ischaemic heart disease $(n=337)$. Outcome measures The Montreal cognitive assessment (MoCA) was obtained. Subclinical LVD was defined as echocardiographic global longitudinal strain $\leq 16 \%$, diastolic dysfunction or LV hypertrophy; abnormal LA reservoir strain (LARS) was defined as $<24 \%$. Subclinical AF was detected using a single-lead portable electrocardiographic device in those without pre-existing AF who gave consent $(n=293)$.

Results Subclinical LVD was found in 155 (46\%), abnormal LARS in 9 (3.6\%) and subclinical AF in 11 (3.8\%). MoCA score consistent with $\mathrm{MCl}(<26)$ was found in $101(30 \%)$; executive function (69\%) and delayed recall $(93 \%)$, were the most frequently abnormal domains. Compared with normal cognition, $\mathrm{MCl}$ was associated with non-adherence to AF screening ( $25 \%$ vs $40 \%, p=0.01$ ). In multivariable logistic regression modelling, educational achievement, systolic blood pressure, body mass index and waist-to-hip ratio were independently associated with $\mathrm{MCl}$. However, neither subclinical AF nor any measure of cardiac dysfunction, were associated with $\mathrm{MCl}$.

Conclusions The $30 \%$ prevalence of $\mathrm{MCl}$ among elderly subjects with risk factors for subclinical LVD and AF has important implications for screening strategies and management. However, $\mathrm{MCl}$ is not associated with subclinical myocardial dysfunction nor subclinical AF. Trial registration number Australian New Zealand Clinical Trials Registry (ACTRN12617000116325).

\section{INTRODUCTION}

Mild cognitive impairment (MCI) describes objective evidence of cognitive impairment
Strengths and limitations of this study

- A community-based study representative of a cardiovascular screening population.

- A validated and easily applied cognitive assessment was used.

- The most sensitive measures of left ventricular (LV) and atrial function were evaluated.

- A longitudinal design would have provided additional insights into impact of subclinical LV dysfunction and subclinical atrial fibrillation on incident cognitive impairment.

Brain MRI may have provided mechanistic insight.

(CI) without significant compromise to independent functioning. ${ }^{1}$ It is a prelude to dementia-a major contributor to mortality and morbidity in our ageing population. ${ }^{2}$ Heart failure (HF) and atrial fibrillation (AF) increase risk of $\mathrm{CI},{ }^{34}$ with between $54 \%$ and $74 \%$ of HF patients affected. ${ }^{5}$ Furthermore, MCI in HF compromises self-management and leads to worse outcomes. ${ }^{6}$ Early detection and prevention of $\mathrm{HF}$ and AF may consequently serve to reduce the burden of MCI. Trials evaluating screening for subclinical left ventricular (LV) dysfunction (LVD) and AF, should incorporate cognitive assessment, not only to inform future screening and prevention strategies but to elucidate clinical associations and mechanisms.

CI in HF is associated with medial temporal lobe atrophy and lower cerebral grey matter volume on neuroimaging, ${ }^{78}$ changes that are more marked compared with those with risk factors but without HF. Whether this is the case in the subclinical phase of HF failure, that is, LVD without HF symptoms, is uncertain. Limited data suggest subclinical LVD is independently associated with MCI. ${ }^{9}$ In addition, reduced systolic function assessed by 
global longitudinal strain (GLS) has been associated with silent cerebral infarcts (SCIs), independent of vascular risk factors. ${ }^{10}$ Left atrial (LA) enlargement has been linked with MCI but this does not appear independent of AF, particularly in longitudinal analyses. ${ }^{11}$ AF may exert its effect on cognitive function via SCIs, presumably due to cardiogenic embolism. The impact of subclinical AF (asymptomatic AF, unrecognised without screening) or LA function on cognition are unknown.

Should screening programmes for subclinical HF and AF be advocated, the cognitive status of the target population must be quantified to inform effective programme design and implementation. Furthermore, the presence of an independent link between subclinical LV and LA dysfunction, subclinical AF and CI remains unclear. Accordingly, assessment of cognitive function was undertaken at baseline in participants enrolled in the Victorian Study of Echocardiographic detection of Subclinical Left Ventricular Dysfunction (Vic-ELF) to (a) establish prevalence and profile of MCI in this population and (b) identify associations between MCI and LV function, LA function and subclinical AF.

\section{METHODS}

\section{Study population}

All subjects were participants in the Vic-ELF trial. Baseline data were used for this cross-sectional substudy. Subjects were recruited from the community via primary care and advertising. Those who were asymptomatic and $\geq 65$ years with hypertension (self-reported, on medication or systolic blood pressure $(\mathrm{SBP}) \geq 140 / 90 \mathrm{~mm} \mathrm{Hg}$ ), type II diabetes mellitus or obesity (body mass index (BMI) $\geq 30 \mathrm{~kg} / \mathrm{m}^{2}$ ) were eligible for inclusion. Those with a history or symptoms of HF or ischaemic heart disease (based on existing clinical indication for echocardiography), LV ejection fraction $\leq 40 \%,>$ moderate valvular disease or oncological life expectancy $<1$ year were excluded.

\section{Patient and public involvement}

Patients were not involved in study design and no evaluation of patient involvement burden was undertaken. All participants will receive information regarding the impact of the research findings after study conclusion.

\section{Clinical assessment}

Comprehensive medical and medication history were taken along with clinical examination. Heart rate, resting averaged blood pressures, BMI, waist and hip circumference and serum N-terminal pro-brain natriuretic peptide (NT-proBNP) were recorded along with a six-minute walk test (6MWT) to assess functional capacity, in accordance with standard procedure. ${ }^{12}$ Patient-reported functional capacity was assessed using the Duke Activity Score Index, which has shown good correlation with peak oxygen uptake, and is readily expressed in metabolic equivalents, a metric familiar to most cardiologists. Health-related quality of life, depression and anxiety were evaluated with the EQ-5D-5L, Generalized Anxiety Disorder 7-Item Scale and the Patient Health Questionnaire-9, respectively. Habitual physical activity was measured $(n=201)$ using waist-worn accelerometers (ActiLife, ActiGraph, Pensacola, Florida, USA) for 7 days. Recordings of less than 4 days were excluded, leaving a total of 190 suitable for analysis.

\section{Cognitive assessment}

The Montreal cognitive assessment (MoCA) was conducted in accordance with instructions. ${ }^{13}$ In brief the MoCA is a short (10-12 min) office-based assessment that evaluates the cognitive domains of executive and visuospatial function; attention, concentration and working memory; short-term memory, language skills and orientation (online supplemental material 1). It is validated in ages 55-85 years and is the preferred screening tool for MCI. ${ }^{14}$ MCI is diagnosed by a score of $<26 / 30$. Graded severity levels of $18-25,10-17$ and $<10$, are suggested for mild, moderate and severe CI, respectively, although supportive data are lacking. Therefore, all CI will be referred to as MCI. A deficit in a domain is defined herein as $\geq 1$ point loss in that domain. MoCA result was unknown to the investigator (SR) evaluating subclinical $\mathrm{AF}$ and atrial function.

\section{Echocardiography}

Resting two-dimensional and Doppler echocardiography was performed with standard equipment (ACUSON SC2000, Siemens Healthcare USA, Mountain View, California, USA) and transducer (4V1c, $1.25-4.5 \mathrm{MHz}$; 4Z1c, 1.5-3.5 MHz) in accordance with guidelines. ${ }^{15} \mathrm{~A}$ vectorvelocity imaging algorithm (Syngo VVI, Siemens Medical Solutions, Siemens Healthcare USA, Mountain View, California, USA) was used for GLS quantification and averaged from apical, 2-chamber, 3-chamber and 4-chamber views. Diastolic function was assessed by measuring mitral inflow peak early diastolic velocity (E), peak late diastolic velocity (A), E/A ratio, septal and lateral mitral annular early diastolic velocities $\left(\mathrm{e}^{\prime}\right)$ and $\mathrm{E} / \mathrm{e}^{\prime}$ ratio. Biplane method of disks (Simpson's modified rule) was used for LA volume quantification and indexed to body surface area (LAVI). Diastolic dysfunction was diagnosed using current recommendations. ${ }^{16} \mathrm{LV}$ mass (LVM) was calculated using the two-dimensional linear method and indexed to body surface area. LVH was defined as LVMI (LVM indexed to body surface area) $95 \mathrm{~g} / \mathrm{m}^{2}$ in women, $115 \mathrm{~g} / \mathrm{m}^{2}$ in men. Subclinical LVD was defined as presence of GLS $\leq 16 \%$, diastolic dysfunction (DD) or LVH.

LA reservoir strain (LARS) measures passive LA stretch during LA filling and is associated with diastolic dysfunction grade, may improve diastolic assessment and is independently predicts incident HF. ${ }^{17-19}$ LARS was assessed by speckle-tracking using a third-party software program (TomTec-Arena (V.TTA2), Tomtec, Munich, Germany). Apical four and 2-chamber images were selected with a frame rate of 60-80 frames/s. The endocardial border of the LA was manually traced, and strain analysis performed 
using the LV strain algorithm, with the average of both the 4-chamber and 2-chamber values. The reference point for image analysis was taken at the onset of the QRS complex (R-R gating). Abnormal LARS was defined as $<24 \%$.

\section{AF screening and echocardiographic risk markers for AF}

Participants without a history of AF or flutter were asked to provide separate consent $(n=293)$. Screening for subclinical AF was performed using a portable, singlelead ECG device (Remon RM-100; Semacare, Beijing, China) using three finger contact electrodes. Recordings lasted $60 \mathrm{~s}$ and were undertaken three times per day for 2 weeks (ie, 42 recordings). Instructions were given verbally face-to-face and in written form. Battery failure, device malfunction or problems relating to dexterity were recorded. ECG recordings were exported as PDF files for interpretation, and all were assessed by a physician. The presence of $\mathrm{AF}$ was defined as a continuous episode of an irregular rhythm $\geq 30$ s with a variable R-R interval and absent $\mathrm{P}$ waves.

A stepwise risk stratification tool for AF using GLS, LAVI and LARS has been devised. ${ }^{20}$ GLS $>14.3 \%$ determines low risk; GLS $<14.3 \%$ and $\mathrm{LAVI}>39 \mathrm{~mL} / \mathrm{m}^{2}$ determines high risk; GLS $<14.3 \%$ and $\mathrm{LAVI} \leq 39 \mathrm{~mL} / \mathrm{m}^{2}$ determines intermediate risk, which can be reclassified to intermediatehigh if LARS $<33.9 \%$. Participants were dichotomised by low/intermediate or high (including intermediate-high) risk based on these criteria. Association between this risk assessment with MCI was assessed individually and combined with detected subclinical AF that is, a group combining those at high risk of subclinical AF plus those with detected subclinical AF.

\section{Statistical analysis}

Continuous variables are presented as median with IQRs or mean $\pm \mathrm{SD}$, based on distribution testing using the Shapiro-Wilk test. Categorical variables are presented as frequencies and percentages. Differences between two independent groups were determined using $\chi^{2}$ and unpaired Student's t-test for categorical and continuous variables, respectively. Variables with a $\mathrm{p}$ value $<0.1$ in univariable analysis were selected for inclusion in multivariable logistic regression modelling. Effect sizes are expressed as ORs with 95\% CIs. Statistical significance was defined as a two-tailed $\mathrm{p}$ value $<0.05$. Analyses were conducted using STATA V.15.1 (StataCorp).

\section{RESULTS}

\section{Participant characteristics}

Of the 337 subjects (age 70 years (IQR 68-73), 58\% women), 292 (87\%) had hypertension with a median duration of 13 years, 108 (32\%) had type II diabetes mellitus with a median duration of 8 years and $214(64 \%)$ were obese (table 1). The majority (62\%) were dyslipidaemic, a significant proportion were current or ex-smokers $(45 \%)$ and a small proportion had a history of stroke or transient ischaemic attack (6\%) and alcohol abuse (7\%). On average, the group spent $66 \%$ of waking time sedentary with levels of moderate-vigorous physical activity (MVPA) falling well below guideline recommendations. Serum NT-proBNP was, on average, in the low-risk range, that is, $<125 \mathrm{pg} / \mathrm{mL}(51 \mathrm{pg} / \mathrm{mL}$ (IQR 30-100)).

\section{Characteristics of $\mathrm{Cl}$ and relation to LV function}

With regards cognitive assessment by MoCA, 101 (30\%) exhibited MCI with an overall average MoCA score of 27 (IQR 25-29). Of the 101 participants with MCI, severity staging showed none with severe CI and only 3 with moderate CI thus the majority had MCI corresponding to a MoCA score between 18 and 25. Overall, delayed recall and executive function had the highest proportion of deficits (237 (70\%) and 145 (43\%), respectively (table 2). There were no differences in the proportion of cognitive domain deficits between those with and without subclinical LVD, except for orientation, although only $2 \%$ of participants had deficits in this domain (table 2).

\section{Subclinical AF screening and $\mathrm{Cl}$}

Of the 293 screened, there were 10 instances of device malfunction leaving 283 for analysis. Subclinical AF was detected in $11(3.9 \%)$. Subclinical AF was equally incident in those with and without MCI, as was pre-existing AF (table 1). In those with pre-existing AF, only 13 (57\%) were taking an anticoagulant. By echocardiographic AF risk stratification, $9(3 \%)$ were deemed high risk and again there was no association with MCI (table 1). MCI was significantly associated with a reduced number of recordings (<30 recordings), $51(25 \%)$ and $33(40 \%)$ for no MCI and MCI, respectively, $\mathrm{p}=0.01$. Therefore, in those undergoing AF screening with a hand-held device a $12 \%(33 / 283)$ rate of non-adherence, related to MCI, was observed.

\section{Clinical and echocardiographic associations with $\mathrm{CI}$}

Those with MCI were less obese and reported significantly fewer years of formal education (table 1). There was a non-significant trend towards higher blood pressure and longer duration of a diagnosis of hypertension and type II diabetes. The proportion with at least moderate anxiety or depression did not differ by presence of MCI, and while on average functional capacity by $6 \mathrm{MWT}$ and minutes per week of MVPA were less in those with MCI, neither were statistically significant (table 1 ). Overall, 155 (46\%) had subclinical LVD. Echocardiographic markers of systolic and diastolic LV function did not differ by presence of MCI (table 3). However, LVMI was significantly higher in those with MCI compared with normal cognition $\left(75 \mathrm{~g} / \mathrm{m}^{2}\right.$ (IQR $\left.60-84\right)$ vs $67 \mathrm{~g} / \mathrm{m}^{2}$ (IQR 55-79), $\mathrm{p}=0.04$, respectively), although this did not translate into a greater proportion of those with MCI having LVH (7 $(7 \%)$ vs $13(5.5 \%), \mathrm{p}=0.62$, respectively). LA function measured by LARS was abnormal $(<24 \%)$ in $9(3.6 \%)$ with a mean value of $36.2 \% \pm 7 \%$. LARS did not differ by presence of MCI, nor did the proportion of those with abnormal LARS (table 3). 
Table 1 Clinical, anthropometric, functional and physical activity measures by presence or absence of mild cognitive impairment (MCl)

\begin{tabular}{|c|c|c|c|c|}
\hline & All $(n=337)$ & No $\mathrm{MCl}(\mathrm{n}=236)$ & $M C I(n=101)$ & $P$ value \\
\hline Age, years (IQR) & $70(68-73)$ & $70(68-73)$ & $70(67-73)$ & 0.83 \\
\hline Gender, female (\%) & $194(58)$ & $140(59)$ & $54(54)$ & 0.32 \\
\hline Hypertension (\%) & $292(87)$ & $201(85)$ & $91(90.1)$ & 0.22 \\
\hline Hypertension duration, years (IQR) & $13(7-20)$ & $12(7-20)$ & $15(7-20)$ & 0.56 \\
\hline Type II diabetes (\%) & $108(32)$ & $72(31)$ & $36(36)$ & 0.36 \\
\hline Diabetes duration, years (IQR) & $8(5-15)$ & $7(4.5-12.5)$ & $10(5-18)$ & 0.1 \\
\hline Obesity (\%) & $214(64)$ & $158(68)$ & $56(56)$ & 0.04 \\
\hline Dyslipidaemia (\%) & $208(62)$ & $145(62)$ & $63(62)$ & 0.9 \\
\hline Ever smoker (\%) & $152(45)$ & $110(47)$ & $42(42)$ & 0.34 \\
\hline AF, known (\%) & $23(7)$ & $14(6)$ & $9(9)$ & 0.32 \\
\hline $\mathrm{AF}$, detected by screening ${ }^{*}(\%)$ & $11(4)$ & $8(4)$ & $3(4)$ & 0.88 \\
\hline High risk for AF† (\%) & $9(3)$ & $8(3)$ & $1(1)$ & 0.21 \\
\hline Stroke/TIA & $21(6)$ & $11(5)$ & $10(10)$ & 0.07 \\
\hline Alcohol abuse (\%) & $25(7)$ & $21(9)$ & $4(4)$ & 0.12 \\
\hline ACE-I/ARB (\%) & $264(78)$ & $183(78)$ & $81(80)$ & 0.59 \\
\hline Beta blocker (\%) & $37(11)$ & $22(9)$ & $15(15)$ & 0.14 \\
\hline Statin (\%) & $179(53)$ & $123(52)$ & $56(55)$ & 0.58 \\
\hline Antiplatelet agent (\%) & $68(20)$ & $43(18)$ & $25(25)$ & 0.17 \\
\hline Anticoagulant (\%) & $16(5)$ & $10(4)$ & $6(6)$ & 0.5 \\
\hline Education, years (IQR) & $12(10-15)$ & $12(10-15)$ & $11(10-14)$ & 0.02 \\
\hline PHQ-9>6 (moderate depression) & $27(8)$ & $20(8.5)$ & $7(6.9)$ & 0.63 \\
\hline GAD-7>6 (moderate anxiety) & $26(8)$ & $19(8)$ & $7(6.9)$ & 0.72 \\
\hline EQ-5D-L score (IQR) & $1(0-2)$ & $1(0-2)$ & $1(0-2)$ & 0.77 \\
\hline Systolic BP, mm Hg (IQR) & $138(131-150)$ & 137 (129-149) & $141(133-151)$ & 0.07 \\
\hline Diastolic BP, mm Hg (IQR) & $83(78-90)$ & $83(77-89)$ & $85(79-91)$ & 0.09 \\
\hline $\mathrm{BMI}, \mathrm{kg} / \mathrm{m}^{2}(\mathrm{IQR})$ & $31(28-35)$ & $32(28-36)$ & $30(27-33)$ & 0.002 \\
\hline Waist-hip ratio (SD) & $0.93(0.09)$ & $0.92(0.09)$ & $0.94(0.09)$ & 0.07 \\
\hline Duke activity score index (IQR) & $51.7(46.7-52.7)$ & $52(49.5-52.7)$ & $50.7(46-52.7)$ & 0.39 \\
\hline Six-minute walk test, m (IQR) & $441(403-476)$ & 445 (403-477) & $438(405-472)$ & 0.49 \\
\hline MVPA, min/week (IQR) & $63(18-144)$ & $65(18-135)$ & $48(17-152)$ & 0.89 \\
\hline Sedentary time, \% (SD) & $66(10)$ & $67(10)$ & $64(9)$ & 0.15 \\
\hline NT-proBNP, pg/mL (IQR) & $51(30-100)$ & $55(31-101)$ & $49(24-95)$ & 0.34 \\
\hline
\end{tabular}

*Total screened $=293$.

†Echocardiographic criteria.

ACE-I/ARB, ACE inhibitor/angiotensin receptor blocker; AF, atrial fibrillation; BMI, body mass index; BP, blood pressure; GAD-7, Generalized Anxiety Disorder 7-Item Scale; MVPA, moderate-vigorous physical activity; NT-proBNP, N-terminal pro-brain natriuretic peptide; PHQ-9,

Patient Health Questionnaire-9; TIA, transient ischaemic attack.

In univariable logistic regression modelling, no echocardiographic markers of LV or LA function, nor presence of AF showed an association with MCI (table 4). Prior cerebrovascular accident, education duration, SBP, BMI and waist-to-hip ratio (WHR) were associated with MCI $(p<0.1)$ (table 4$)$. In multivariable analysis, MCI was independently associated with higher SBP (OR 1.02 (1.00-1.04), p=0.03) and WHR (OR 40 (2.3$708), \mathrm{p}=0.01$ ), while greater numbers of years in formal education $(0.9$ (0.86-0.98), $\mathrm{p}=0.01)$ and higher BMI $(0.9$ $(0.85-0.95), \quad \mathrm{p}<0.001)$ were independently associated with normal cognition.

\section{DISCUSSION}

Up to $30 \%$ of individuals included in a screening programme for subclinical LVD and AF had MCI, manifest most commonly as executive dysfunction, and poor 
Table 2 Mild cognitive impairment (MCl) and deficits in individual cognitive domains according to presence or absence of subclinical left ventricular dysfunction (LVD)

\begin{tabular}{llllll}
\hline & $\begin{array}{l}\text { Overall } \\
(\mathbf{n}=\mathbf{3 3 7})\end{array}$ & $\mathbf{M C l}(\mathbf{n = 1 0 1 )})$ & $\begin{array}{l}\text { Normal LV function } \\
(\mathbf{n}=\mathbf{1 7 5})\end{array}$ & $\begin{array}{l}\text { Subclinical LVD } \\
(\mathbf{n = 1 6 2})\end{array}$ & P value \\
\hline MCl (MoCA<26) & $101(30)$ & & $52(29.7)$ & $49(30.2)$ & 0.9 \\
\hline Moderate Cl (MoCA <18) & $3(3)$ & & $2(4)$ & $1(2)$ & 0.7 \\
\hline Executive and visuospatial (\%) & $145(43)$ & $70(69)$ & $75(43)$ & $70(43)$ & 0.9 \\
\hline Naming (\%) & $15(4.5)$ & $9(9)$ & $6(3.4)$ & $9(5.6)$ & 0.34 \\
\hline Attention (\%) & $5(1.5)$ & $5(5)$ & $4(2.3)$ & $1(0.62)$ & 0.21 \\
\hline Language (\%) & $124(37)$ & $69(68)$ & $70(40)$ & $54(33)$ & 0.2 \\
\hline Abstraction (\%) & $88(26)$ & $61(60)$ & $50(29)$ & $38(23)$ & 0.29 \\
\hline Delayed recall (\%) & $237(70)$ & $94(93)$ & $121(69)$ & $116(72)$ & 0.62 \\
\hline Orientation (\%) & $7(2)$ & $6(6)$ & $7(4)$ & $0(0)$ & 0.01 \\
\hline
\end{tabular}

$P$ value for comparison of normal left ventricular function versus subclinical LVD.

MoCA, Montreal cognitive assessment.

recall of recently delivered information. This is more prevalent than in unselected people aged $>65$ years, among whom the prevalence of MCI is $3 \%-19 \% .^{21}$ The higher prevalence in our population supports the notion that MCI can be expected in people at risk of $\mathrm{HF}$ and $\mathrm{AF}$. This is consistent with evidence that cardiovascular risk factors compromise executive function, which is especially true for hypertension-even at subclinical levels. ${ }^{22}$

For the first time, associations were sought between sensitive deformation markers of LV and LA function (strain) and none were found, nor did we find evidence that subclinical AF or high AF risk was associated with MCI, although the number of subjects concerned was low. However, consistent with existing data, lower educational achievement, higher SBP and visceral adiposity, but lower BMI were independently associated with $\mathrm{MCI}^{23} 24$ (figure 1). If an independent association exists between $\mathrm{HF}$ and CI, then our data suggest this is not apparent in the subclinical phase of HF.

\section{Cognition and cardiac disease}

There is contemporary focus on cognitive dysfunction in the setting of cardiac diseases, principally $\mathrm{HF}$ and AF. CI, specifically vascular CI shares well documented risk factors with HF and AF. Exposure to hypertension, diabetes, smoking and abdominal obesity in mid-life is associated with an accelerated decline in executive function a decade later. This is coupled with MRI evidence of cerebral vascular damage and atrophy. ${ }^{24}$

Table 3 Echocardiographic variables by presence or absence of mild cognitive impairment (MCl)

\begin{tabular}{|c|c|c|c|}
\hline & No $\mathrm{MCl}(\mathrm{n}=236)$ & $\mathrm{MCl}(\mathrm{n}=101)$ & $P$ value \\
\hline LV ejection fraction, \% (SD) & $62(6.8)$ & $62(5.8)$ & 0.7 \\
\hline GLS, \% (IQR) & $18.7(17-20)$ & $18.7(17-20)$ & 0.87 \\
\hline E/A ratio (IQR) & $0.8(0.68-0.95)$ & $0.82(0.69-0.99)$ & 0.63 \\
\hline $\mathrm{e}^{\prime}, \mathrm{cm} / \mathrm{s}(\mathrm{IQR})$ & $7.5(6.3-8.9)$ & $7.5(6.5-8.7)$ & 0.67 \\
\hline $\mathrm{E} / \mathrm{e}^{\prime}(\mathrm{IQR})$ & $8.2(6.9-10.2)$ & $8.7(7.2-11)$ & 0.32 \\
\hline LAVI, $\mathrm{mL} / \mathrm{m}^{2}(\mathrm{IQR})$ & $34(28-40)$ & $33(29-42)$ & 0.56 \\
\hline LA reservoir strain*, \% (SD) & $36.2(7)$ & $36.1(7)$ & 0.9 \\
\hline LARS $<24 \% *(\%)$ & $7(4)$ & $2(3)$ & 0.61 \\
\hline Relative wall thickness (IQR) & $0.37(0.34-0.43)$ & $0.39(0.33-0.43)$ & 0.96 \\
\hline LV mass indexed, $\mathrm{g} / \mathrm{m}^{2}$ (IQR) & $67(55-79)$ & $75(60-84)$ & 0.04 \\
\hline Subclinical LV dysfunction (\%) & $113(48)$ & $49(48.5)$ & 0.9 \\
\hline Systolic dysfunction (GLS $\leq 16 \%)$ & $42(18)$ & $13(13)$ & 0.26 \\
\hline Diastolic dysfunction (\%) & $54(23)$ & $26(26)$ & 0.54 \\
\hline LV hypertrophy (\%) & $13(5.5)$ & $7(7)$ & 0.62 \\
\hline
\end{tabular}

${ }^{*}$ Available in 248 participants.

GLS, global longitudinal strain; LARS, left atrial reservoir strain; LAVI, left atrial volume indexed to body surface area; LV, left ventricular. 
Table 4 Logistic regression modelling for prediction of mild cognitive impairment

\begin{tabular}{|c|c|c|c|c|}
\hline & \multicolumn{2}{|l|}{ Univariable } & \multicolumn{2}{|l|}{ Multivariable } \\
\hline & OR $(95 \% \mathrm{Cl})$ & $P$ value & OR $(95 \% \mathrm{Cl})$ & $P$ value \\
\hline Age, years & 1.00 (0.95 to 1.06$)$ & 0.88 & & \\
\hline Female gender & $0.8(0.49$ to 1.26$)$ & 0.32 & & \\
\hline Hypertension & 1.58 (0.75 to 3.33$)$ & 0.23 & & \\
\hline Hypertension duration & 1.00 (0.97 to 1.03$)$ & 0.76 & & \\
\hline Type II diabetes & 1.26 (0.77 to 2.06$)$ & 0.36 & & \\
\hline Diabetes duration & 1.03 (0.98 to 1.09$)$ & 0.22 & & \\
\hline Dyslipidaemia & 1.03 (0.63 to 1.66$)$ & 0.9 & & \\
\hline Ever smoker & 0.88 (0.23 to 3.44$)$ & 0.86 & & \\
\hline Stroke/TIA & 2.2 (0.87 to 5.6$)$ & 0.09 & 2.5 (0.93 to 6.8$)$ & 0.07 \\
\hline AF (known) & 1.54 (0.65 to 3.69$)$ & 0.33 & & \\
\hline AF (detected or high risk) & 0.63 (0.2 to 1.96$)$ & 0.43 & & \\
\hline Education, years & 0.92 (0.86 to 0.98$)$ & 0.02 & 0.9 (0.86 to 0.98$)$ & 0.01 \\
\hline Depression (PHQ-9>6), \% & 0.8 (0.32 to 2$)$ & 0.6 & & \\
\hline Anxiety (GAD-7>6), \% & 0.85 (0.33 to 2.1$)$ & 0.72 & & \\
\hline ACE-I/ARB & 1.17 (0.65 to 2$)$ & 0.59 & & \\
\hline Beta blocker & $1.7(0.84$ to 3.4$)$ & 0.14 & & \\
\hline Statin & 1.14 (0.7 to 1.8$)$ & 0.58 & & \\
\hline Antiplatelet & 1.47 (0.84 to 2.59$)$ & 0.17 & & \\
\hline Anticoagulant & $1.43(0.5$ to 4$)$ & 0.5 & & \\
\hline Systolic BP, mm Hg & $1.02(0.99$ to 1.03$)$ & 0.07 & $1.02(1.00$ to 1.04$)$ & 0.03 \\
\hline Diastolic BP, mm Hg & 1.02 (0.99 to 1.04$)$ & 0.2 & & \\
\hline $\mathrm{BMI}, \mathrm{kg} / \mathrm{m}^{2}$ & 0.93 (0.88 to 0.97$)$ & 0.001 & 0.9 (0.85 to 0.95$)$ & $<0.001$ \\
\hline Waist-hip ratio & 11 (0.8 to 161$)$ & 0.07 & 40 (2.3 to 708$)$ & 0.01 \\
\hline NT-proBNP, pg/mL & 0.99 (0.99 to 1.00$)$ & 0.7 & & \\
\hline MVPA, hour/week & 0.99 (0.99 to 1.00$)$ & 0.98 & & \\
\hline Sedentary time, \% & $0.98(0.94$ to 1.01$)$ & 0.15 & & \\
\hline \multicolumn{5}{|l|}{ Echocardiographic classifications } \\
\hline Subclinical LV dysfunction & $1.03(0.64$ to 1.63$)$ & 0.9 & & \\
\hline Systolic dysfunction (GLS $\leq 16 \%$ ) & 0.68 (0.35 to 1.33$)$ & 0.26 & & \\
\hline Diastolic dysfunction & 1.18 (0.69 to 2$)$ & 0.54 & & \\
\hline LV hypertrophy & 1.27 (0.49 to 3.3$)$ & 0.62 & & \\
\hline \multicolumn{5}{|c|}{ Echocardiographic continuous measures } \\
\hline LV ejection fraction, \% & 0.99 (0.95 to 1.03$)$ & 0.7 & & \\
\hline GLS, \% & $1.01(0.92$ to 1.11$)$ & 0.82 & & \\
\hline $\mathrm{e}^{\prime}, \mathrm{cm} / \mathrm{s}$ & 0.96 (0.84 to 1.09$)$ & 0.57 & & \\
\hline$E / e^{\prime}$ & $1.03(0.94$ to 1.12$)$ & 0.4 & & \\
\hline LAVI mL/m² & 1 (0.98 to 1.03$)$ & 0.56 & & \\
\hline LA reservoir strain, \% & $0.98(0.96$ to 1.04$)$ & 0.91 & & \\
\hline LARS $<24 \%$ & $0.66(0.13$ to 3.27$)$ & 0.61 & & \\
\hline LV mass indexed, $\mathrm{g} / \mathrm{m}^{2}$ & 1.00 (0.99 to 1.02$)$ & 0.13 & & \\
\hline
\end{tabular}

ACE-I/ARB, ACE inhibitor/angiotensin receptor blocker; AF, atrial fibrillation; BMI, body mass index; BP, blood pressure; GAD-7, Generalized Anxiety Disorder 7-Item Scale; GLS, global longitudinal strain; LA, left atrial; LARS, left atrial reservoir strain; LAVI, left atrial volume indexed to body surface area; LV, left ventricular; MVPA, moderate-vigorous physical activity; NT-proBNP, N-terminal pro-brain natriuretic peptide; PHQ9, Patient Health Questionnaire-9; TIA, transient ischaemic attack. 
$30 \%$ prevalence of mild cognitive impairment

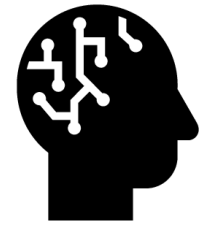

Implications for screening

- $1.6 \times$ more likely to be non-adherent to handheld AF screening

- Consideration of strategies to optimise engagement e.g. wearables, family involvement

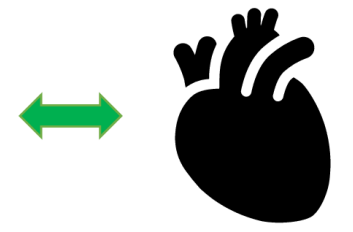

Associations with clinical factors and cardiac function

- No association with subclinical AF

- No association with LV global longitudinal strain or diastolic parameters

- No association with left atrial reservoir strain

- Systolic blood pressure, educational attainment, body mass index and abdominal adiposity are independently associated

Figure 1 Summary of study findings. AF, atrial fibrillation; LV, left ventricular.

Symptomatic HF is independently associated CI, although data with robust adjustment for shared risk factors is sparse. Nevertheless, the impact is significant, with most recent estimates of incidence being around $30 \%$ over 3.5 years, ${ }^{25}$ with cerebral hypoperfusion and subclinical cardiogenic emboli likely mechanisms. ${ }^{26}$ Population studies demonstrate conflicting results regarding associations between LV function and cognition. Cross-sectional data from the Framingham Heart Study found a U-shaped relationship between LVEF quintiles and cognition with the extremes displaying worse cognitive performance (memory and executive function) ${ }^{27}$ Conversely, longitudinal data from the Netherlands demonstrated that LAVI but not LVEF at baseline was associated with lower performances in attention and executive function at follow-up. ${ }^{28}$ Furthermore, another cross-sectional population study found lower systolic function, assessed by tissue Doppler early systolic peak velocity, was not associated with poor cognitive performance but was associated with lower total brain volume. ${ }^{29}$ With regards LA size, several studies have demonstrated an association between greater LA size and CI by global assessment or specific domain testing. ${ }^{6} 2829$ However, adjustment for $\mathrm{AF}$ is inconsistent and recent evidence suggests the association is not independent of known AF. ${ }^{11}$

Less is known about the link between cardiac dysfunction and cognition in asymptomatic patients. In patients with chronic heart disease (eg, coronary disease) but without symptomatic HF, diastolic filling pressure estimated by $\mathrm{E} / \mathrm{e}^{\prime}$, was associated with significantly higher odds of MCI after comprehensive adjustment for clinical factors, although effect size was small (OR 1.07, 1.01-1.13, $\mathrm{p}=0.022) .{ }^{9}$ This finding did not extend to LVMI, LAVI or stroke volume index. ${ }^{9}$ In a population without symptomatic cardiac or cerebrovascular disease, those with SCIs on
MRI had significantly lower systolic function, as assessed by GLS. ${ }^{30}$ Moreover, GLS in those with SCIs was in the abnormal range.

$\mathrm{AF}$ is associated with a $42 \%$ increase in risk of dementia, independent of age and cardiovascular risk factors. ${ }^{4} 31$ Interestingly, this association appears strongest in those $<70$ years with data suggesting no association $>67$ years, presumably due to the influence of neurodegenerative pathophysiology. ${ }^{31} 32$ This is significant given the median age in our study was 70 years. The most prominent mechanism behind the association between AF and CI is SCIs, the presence of which determine cognitive decline associated with AF, and conversely those with AF without SCIs do not exhibit cognitive decline. ${ }^{33}$ However, no study has examined the distribution of SCIs preventing inference about the pathophysiological mechanism, that is, small vessel vs embolic disease. Anticoagulation in AF is associated with up to a $60 \%$ reduction in cognitive decline and incident dementia, supporting a cardioembolic mechanism. ${ }^{34}$ Neuroimaging would have strengthened our study and revealed whether those with AF were free of SCIs thus potentially explaining the lack of association with MCI.

\section{Clinical implications}

Clinicians involved in management of patient with $\mathrm{CV}$ risk factors must be alert to the significant proportion of patients who will have MCI-affecting their ability to recall medical information and self-manage aspects of their condition. Indeed, those with MCI progress to dementia at a rate of over $50 \%$ in 5 years. ${ }^{21}$ Our data highlight that even in the early stages of cognitive compromise modifiable risk factors that is, systolic hypertension and abdominal obesity are contributors, and it may be argued that cognitive screening be undertaken routinely 
in this scenario. We did not find evidence of an association between certain echocardiographic measures, even sensitive markers of LV and LA function. So, based on these data, echocardiographic abnormality alone should not prompt cognitive evaluation.

In terms of $\mathrm{HF}$ prevention, while management of subclinical disease largely rests on risk factor control, the onus is on the patient to recognise the often-insidious transition to a symptomatic state. Current American College of Cardiology/American Heart Association HF management guidelines suggest that patients with subclinical HF undertake self-surveillance for symptoms and our data highlight one of the problems with this approach that is, the potential for under-recognition due to CI. While screening for subclinical LVD is not currently advocated, it is plausible that early institution of therapy may preserve cognition if progression to symptomatic HF is delayed or prevented. Indeed, anticoagulation for AF, whether permanent or paroxysmal, is associated with a significant reduction in $\mathrm{CI},{ }^{34}$ an observation that could extend to subclinical AF detected by screening.

One of the primary objectives of this study was to assess the prevalence of MCI and therefore the consequences to delivery of screening programmes for $\mathrm{HF}$ and AF. This study population may have been subject to selection bias given they had sufficient cognition to apply for the trial, meaning the true prevalence is likely higher. However, for those with established dementia, prevention of $\mathrm{HF}$ or $\mathrm{AF}$ is not their primary care goal. Population-based screening for dementia or MCI is not presently advocated, however, a novel proposal may be that HF/AF screening be used as a platform for cognitive screening given the high yield in this cohort. Our data suggest that strategies to optimise engagement and follow-up with an HF/AF screening programme should be considered. For example, engagement of services beyond the screening programme and consideration given to the impact of reduced cognition and health literacy. When cognition is compromised, close relatives can assist with health literacy to promote use of health services. Our finding of a $12 \%$ rate of nonadherence to self-initiated AF screening, that related to MCI, is also of importance in considering the mode of delivery of AF screening. Technologies like monitoring patches or smartwatches may be more effective than devices that participants are required to operate.

\section{Limitations}

The study would have been strengthened by a longitudinal design, to additionally assess impact on incident MCI. While our sample size was not based on calculation, it is comparable to other studies in specific populations. Furthermore, a larger sample size would have yielded more accurate effect sizes. As mentioned previously, brain MRI would have provided additional mechanistic insights. Our method of assessment for MCI was chosen both for its speed and validity. However, use of more detailed tests for individual cognitive domains may have added more depth to our results and made comparisons with other studies easier. Indeed, variation in the literature surrounding CV disease and cognition may be largely due to inconsistencies in methods. Finally, it should be borne in mind that while a significant proportion of subjects exhibited subclinical LVD, the number with reduced atrial function and/or subclinical AF was low, limiting the certainty of our observations.

\section{CONCLUSION}

Elderly subjects enrolled in a trial screening for subclinical LVD and AF exhibited a $30 \%$ prevalence of MCI. There was no association between sensitive measures of $\mathrm{LV}$ and LA function nor subclinical AF and presence of MCI.

\section{Twitter Elizabeth L Potter @DrLizPotter}

Contributors ELP and TM contributed to the conception and design of the work. All authors contributed to the acquisition and interpretation of data. ELP analysed the data and drafted the manuscript. SR and LW critically revised the manuscript. TM obtained funding for the study and critically revised the manuscript. All gave final approval and agree to be accountable for all aspects of work ensuring integrity and accuracy.

Funding The work was partially supported by a Partnership grant (1149692) from the National Health and Medical Research Council, Canberra, the lan Potter Foundation, Melbourne, and the Baker Heart and Diabetes Institute. ELP is supported by a Monash University postgraduate scholarship.

Competing interests None declared.

Patient consent for publication Not required.

Ethics approval The study was approved by a Human Research Ethics Committee (Bellberry, HREC number 2016-10-727) and all participants gave written informed consent.

Provenance and peer review Not commissioned; externally peer reviewed.

Data availability statement Data are available upon reasonable request. Data are availabe upon reasonable request.

Supplemental material This content has been supplied by the author(s). It has not been vetted by BMJ Publishing Group Limited (BMJ) and may not have been peer-reviewed. Any opinions or recommendations discussed are solely those of the author(s) and are not endorsed by BMJ. BMJ disclaims all liability and responsibility arising from any reliance placed on the content. Where the content includes any translated material, BMJ does not warrant the accuracy and reliability of the translations (including but not limited to local regulations, clinical guidelines, terminology, drug names and drug dosages), and is not responsible for any error and/or omissions arising from translation and adaptation or otherwise.

Open access This is an open access article distributed in accordance with the Creative Commons Attribution Non Commercial (CC BY-NC 4.0) license, which permits others to distribute, remix, adapt, build upon this work non-commercially, and license their derivative works on different terms, provided the original work is properly cited, appropriate credit is given, any changes made indicated, and the use is non-commercial. See: http://creativecommons.org/licenses/by-nc/4.0/.

ORCID iD

Elizabeth L Potter http://orcid.org/0000-0002-3789-9959

\section{REFERENCES}

1 Langa KM, Levine DA. The diagnosis and management of mild cognitive impairment: a clinical review. JAMA 2014;312:2551-61.

22019 AloHaW. Hospital care for people with dementia 2016-17. cat no age 94. Canberra: AlHW.

3 Vogels RLC, Scheltens P, Schroeder-Tanka JM, et al. Cognitive impairment in heart failure: a systematic review of the literature. Eur $J$ Heart Fail 2007;9:440-9.

4 Santangeli P, Di Biase L, Bai R, et al. Atrial fibrillation and the risk of incident dementia: a meta-analysis. Heart Rhythm 2012;9:1761-8. 
5 Leto L, Feola M. Cognitive impairment in heart failure patients. $J$ Geriatric Cardiol 2014;11:316-28.

6 Huynh QL, Negishi K, De Pasquale CG, et al. Cognitive domains and Postdischarge outcomes in hospitalized patients with heart failure. Circ Heart Fail 2019;12:e006086.

7 Almeida OP, Garrido GJ, Beer C, et al. Cognitive and brain changes associated with ischaemic heart disease and heart failure. Eur Heart J 2012;33:1769-76.

8 Frey A, Sell R, Homola GA, Homola György A, et al. Cognitive deficits and related brain lesions in patients with chronic heart failure. JACC Heart Fail 2018;6:583-92.

9 Sacre JW, Ball J, Wong C, et al. Mild cognitive impairment is associated with subclinical diastolic dysfunction in patients with chronic heart disease. Eur Heart J Cardiovasc Imaging 2018;19:285-92.

10 Russo C, Jin Z, Homma S, et al. Subclinical left ventricular dysfunction and silent cerebrovascular disease: the cardiovascular abnormalities and brain lesions (CABL) study. Circulation 2013;128:1105-11.

11 Zhang MJ, Norby FL, Lutsey PL, et al. Association of left atrial enlargement and atrial fibrillation with cognitive function and decline: the ARIC-NCS. J Am Heart Assoc 2019;8:e013197.

12 ATS Committee on Proficiency Standards for Clinical Pulmonary Function Laboratories. Ats statement: guidelines for the six-minute walk test. Am J Respir Crit Care Med 2002;166:111-7.

13 . Available: https://www.mocatest.org

14 Abd Razak MA, Ahmad NA, Chan YY, et al. Validity of screening tools for dementia and mild cognitive impairment among the elderly in primary health care: a systematic review. Public Health 2019;169:84-92.

15 Lang RM, Badano LP, Mor-Avi V, et al. Recommendations for cardiac chamber quantification by echocardiography in adults: an update from the American Society of echocardiography and the European association of cardiovascular imaging. J Am Soc Echocardiogr 2015;28:1-39.

16 Nagueh SF, Smiseth OA, Appleton CP, et al. Recommendations for the evaluation of left ventricular diastolic function by echocardiography: an update from the American Society of Echocardiography and the European Association of Cardiovascular Imaging. J Am Soc Echocardiogr 2016;29:277-314.

17 Singh A, Addetia K, Maffessanti F, et al. LA strain for categorization of LV diastolic dysfunction. JACC Cardiovasc Imaging 2017:10:735-43.

18 Morris DA, Belyavskiy E, Aravind-Kumar R, et al. Potential Usefulness and Clinical Relevance of Adding Left Atrial Strain to Left Atrial Volume Index in the Detection of Left Ventricular Diastolic Dysfunction. JACC Cardiovasc Imaging 2018;11:1405-15.
19 Potter EL, Ramkumar S, Kawakami H, et al. Association of asymptomatic diastolic dysfunction assessed by left atrial strain with incident heart failure. JACC Cardiovasc Imaging 2020;13:2316-26.

20 Ramkumar S, Ochi A, Kawakami H, et al. Echocardiographic risk assessment to guide screening for atrial fibrillation. J Am Soc Echocardiogr 2019;32:1259-67.

21 Gauthier S, Reisberg B, Zaudig M, et al. Mild cognitive impairment. Lancet 2006;367:1262-70.

22 Leritz EC, McGlinchey RE, Kellison I, et al. Cardiovascular disease risk factors and cognition in the elderly. Curr Cardiovasc Risk Rep 2011;5:407-12

23 Hou Q, Guan Y, Yu W, et al. Associations between obesity and cognitive impairment in the Chinese elderly: an observational study. Clin Interv Aging 2019;14:367-73.

24 Debette S, Seshadri S, Beiser A, et al. Midlife vascular risk factor exposure accelerates structural brain aging and cognitive decline. Neurology 2011;77:461-8.

25 Lee TC, Qian M, Liu Y, et al. Cognitive Decline Over Time in Patients With Systolic Heart Failure. JACC: Heart Failure 2019;7:1042-53.

26 Cannon JA, McMurray JJ, Quinn TJ. 'Hearts and minds': association, causation and implication of cognitive impairment in heart failure. Alzheimers Res Ther 2015;7:22.

27 Jefferson AL, Himali JJ, Au R, et al. Relation of left ventricular ejection fraction to cognitive aging (from the Framingham heart study). Am J Cardiol 2011;108:1346-51.

28 van den Hurk K, Reijmer YD, van den Berg E, et al. Heart failure and cognitive function in the general population: the Hoorn study. Eur $J$ Heart Fail 2011;13:1362-9.

29 Park CM, Williams ED, Chaturvedi N, et al. Associations between left ventricular dysfunction and brain structure and function: findings from the sabre (Southall and Brent revisited) study. J Am Heart Assoc 2017;6:e004898.

30 Prins ND, van Dijk EJ, den Heijer T, et al. Cerebral white matter lesions and the risk of dementia. Arch Neurol 2004:61:1531-4.

31 de Bruijn RFAG, Heeringa J, Wolters FJ, et al. Association between atrial fibrillation and dementia in the general population. JAMA Neurol 2015;72:1288-94.

32 Marengoni A, Qu C, Winblad B, et al. Atrial fibrillation, stroke and dementia in the very old: a population-based study. Neurobiol Aging 2011:32:1336-7.

33 Chen LY, Lopez FL, Gottesman RF, et al. Atrial fibrillation and cognitive decline-the role of subclinical cerebral infarcts: the Atherosclerosis risk in Communities study. Stroke 2014;45:2568-74.

34 Ding M, Fratiglioni L, Johnell K, et al. Atrial fibrillation, antithrombotic treatment, and cognitive aging: a population-based study. Neurology 2018;91:e1732-40. 